\title{
Patients with impaired fasting glucose exhibit a more frequent non-dipper or riser blood pressure pattern compared with normoglycemic patients
}

Fernández-García, José Carlos (1), Cortés-Salazar, Carmen María (2), de la Cruz-Troca, Juan José (3), García Arnés, Juan Antonio (4), Aranda-Lara, Pedro (5)

1. Endocrinology Department. Virgen de la Victoria University Hospital. Málaga (Spain).

2. Badolatosa Primary Care Centre. Seville (Spain).

3. Department of Preventive Medicine and Public Health, School of Medicine, Universidad Autónoma de Madrid (Spain)

4. Endocrinology Department. Carlos Haya University Hospital. Málaga (Spain).

5. Hypertension Unit. Carlos Haya University Hospital. Málaga (Spain).

\section{Objective}

- To study blood pressure circadian pattern in patients with impaired fasting glucose (IFG) evaluated with ambulatory blood pressure monitoring (ABPM).

\section{Baseline characteristics}

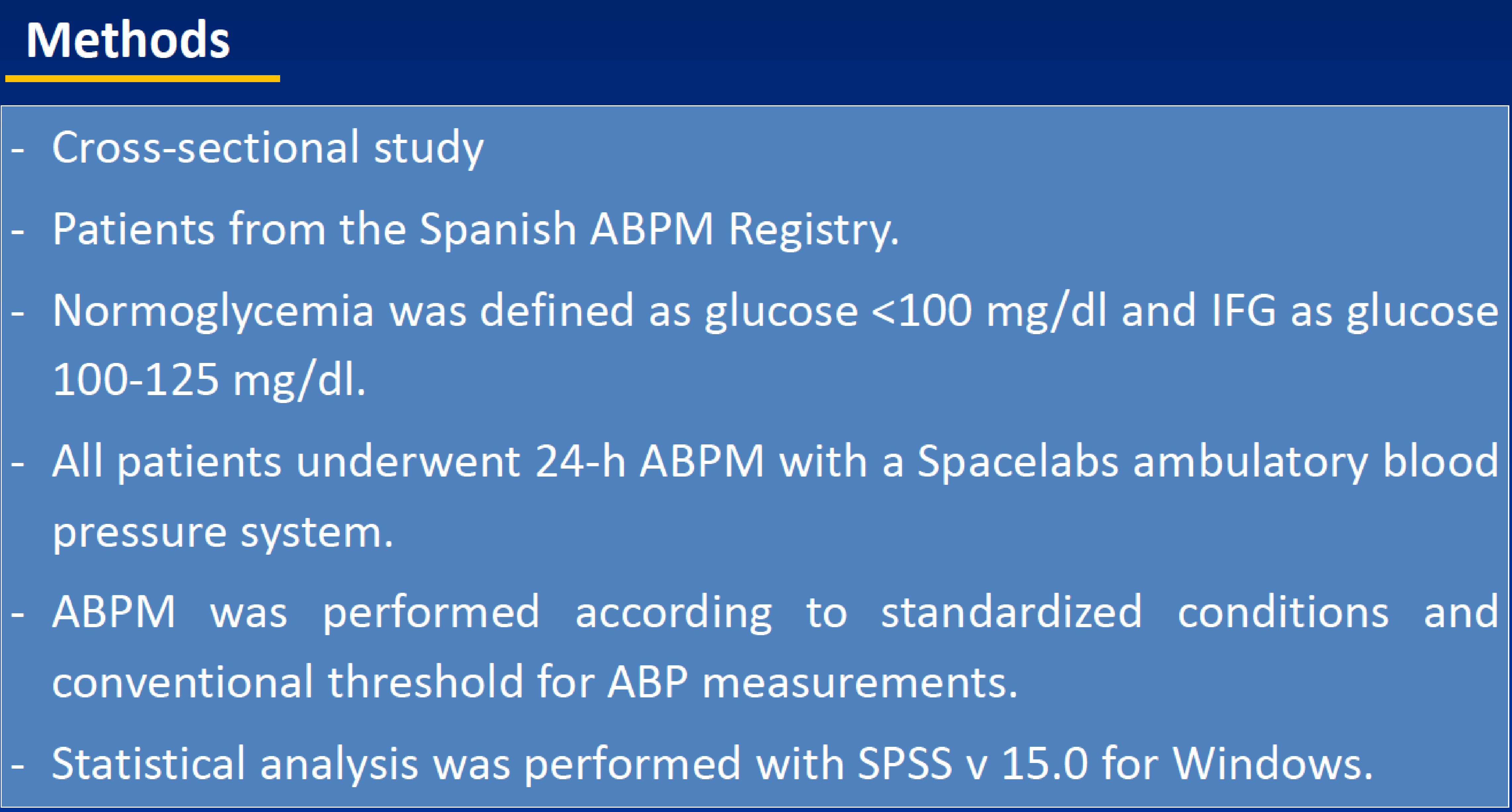

\begin{tabular}{|c|ccc|}
\hline & \multicolumn{3}{|c|}{$\mathrm{n}-24708$} \\
& Normoglycemia & IFG & $\mathrm{p}$ \\
& $(\mathrm{n}-16587,67.2 \%)$ & $(\mathrm{n}-8121,32.8 \%)$ & \\
\hline Age (years) & $56.0 \pm 14.6$ & $60.2 \pm 12.6$ & $<0.001$ \\
Male (\%) & 49.5 & 57.5 & $<0.001$ \\
BMI (kg/m $\left.{ }^{2}\right)$ & $28.2 \pm 4.4$ & $29.6 \pm 4.3$ & $<0.001$ \\
Waist circumference (cm) & & & \\
- Male & $99.2 \pm 10.3$ & $102.4 \pm 10.1$ & $<0.001$ \\
- Women & $92.8 \pm 12.1$ & $97.2 \pm 11.8$ & $<0.001$ \\
Office BP (mmHg) & & & \\
- Systolic & $147.3 \pm 18.7$ & $150.4 \pm 19.2$ & $<0.001$ \\
- Diastolic & $87.8 \pm 11.1$ & $87.7 \pm 12.1$ & $\mathrm{~ns}$ \\
\hline
\end{tabular}

\section{Dipper and non-dipper blood pressure 24-hour patterns}

\begin{tabular}{|cccc|}
\hline \% & IFG & p \\
\hline Dipper & 44.1 & 41.3 & $<0.001$ \\
Extreme Dipper & 7.8 & 7.4 & ns \\
Non-dipper & 37.6 & 39.0 & $<0.001$ \\
Riser & 10.6 & 12.3 & $<0.001$ \\
\hline Non-dipper + Riser & 48.2 & 51.3 & $<0.001$ \\
\hline
\end{tabular}

\section{Conclusions}

4 Patients with IFG are older, more frequently men, and are more obese. These patients show higher $24 \mathrm{~h}$, daytime and nighttime SBP, but lower 24h, daytime and nighttime DBP.

$\Rightarrow$ IFG patients have a higher percentage of non-dipper + riser pattern in the circadian blood pressure evaluation.

\section{ABPM Values}

\begin{tabular}{|c|c|c|c|}
\hline ABPM (mmHg) & Normoglycemia & IFG & $p$ \\
\hline \multicolumn{4}{|l|}{$24 h$} \\
\hline - SBP & $127.9 \pm 13.2$ & $128.9 \pm 13.7$ & $<0.001$ \\
\hline - DBP & $77.4 \pm 10.0$ & $76.4 \pm 10.1$ & $<0.001$ \\
\hline \multicolumn{4}{|l|}{ Daytime } \\
\hline - SBP & $131.3 \pm 13.3$ & $132.2 \pm 14.1$ & $<0.001$ \\
\hline - DBP & $80.6 \pm 10.5$ & $79.3 \pm 10.6$ & $<0.001$ \\
\hline \multicolumn{4}{|l|}{ Nighttime } \\
\hline$-\mathrm{SBP}$ & $118.2 \pm 14.9$ & $119.8 \pm 15.6$ & $<0.001$ \\
\hline - DBP & $68.7 \pm 10.1$ & $68.3 \pm 10.2$ & $<0.001$ \\
\hline \multicolumn{4}{|l|}{ BP 24h } \\
\hline$<130 / 80(\%)$ & 46.6 & 45.8 & ns \\
\hline Daytime BP & & & \\
\hline$<135 / 85(\%)$ & 52.5 & 52.3 & ns \\
\hline Nighttime BP & & & \\
\hline$<120 / 70(\%)$ & 44.8 & 43.2 & 0.022 \\
\hline
\end{tabular}

